\title{
Lumbar-Peritoneal Shunt followed by Donepezil Administration for Residual Cognitive Impairment in Idiopathic Normal Pressure Hydrocephalus: A Case Report
}

Shusuke Moriuchi ${ }^{1 *}$, Makoto Dehara ${ }^{1}$, Takeshi Shimizu${ }^{1}$, Shogo Fukuya ${ }^{1}$, Takanori Fukunaga ${ }^{1}$, Yasushi Hagihara ${ }^{1}$, and Takao Soda ${ }^{2}$

${ }^{1}$ Departments of Neurosurgery, Rinku General Medical Center, Osaka 598-8577, Japan

${ }^{2}$ Neurology, Rinku General Medical Center, Osaka 598-8577, Japan

"Corresponding author: Shusuke Moriuchi, Department of Neurosurgery, Rinku General Medical Center, 2-23 Rinku-Orai Kita, Izumisano-City, Osaka 598-8577, Japan, Tel: 81+72-469-3111; Fax: 81+72-469-7929; E-mail: s-moriuchi@rgmc.izumisano.osaka.jp

Received date: Nov 20, 2014, Accepted date: Mar 11, 2015, Published date: Mar 16, 2015

Copyright: ( 2015 Moriuchi S, et al. This is an open-access article distributed under the terms of the Creative Commons Attribution License, which permits unrestricted use, distribution, and reproduction in any medium, provided the original author and source are credited.

\begin{abstract}
We often encounter idiopathic normal-pressure hydrocephalus (iNPH) and mild cognitive impairment (MCl) in the same patient. Four iNPH patients whose gait improved following placement of a lumbar-peritoneal shunt (LPS) and whose cognitive function improved after administration of donepezil are reported. LPS is useful for improvement of gait disturbance in iNPH, and donepezil is used to treat impaired memory in dementia of Alzheimer's type (DAT). These four patients showed improvements in gait disturbances postoperatively, and just after donepezil administration, improvements in cognitive symptoms were recognized in the memory tests of the Mini-Mental State Examination (MMSE). The four patients (two males and two females) ranged in age from 72 to 81 years (mean age: 77 years). In all patients, disproportionately enlarged subarachnoid-space hydrocephalus (DESH) was recognized on imaging examinations, and improvements after the tap test were considered to be indications for surgery. The improvements in the Timed Up and Go Test (TUG) were from $25 \%$ to $36 \%$. The patients' mean MMSE score was 27 points preoperatively, 27 points at 1 week after LPS placement, and 30 points at 3 months after LPS placement. Treatment with donepezil appeared to be effective for residual cognitive impairments after LPS placement.
\end{abstract}

Keywords: Mild cognitive impairment; Donepezil; Gait disturbance; Lumbar-peritoneal shunt; Normal pressure hydrocephalus

\section{Abbreviations:}

NPH: Normal Pressure Hydrocephalus; LPS: Lumbar-peritoneal Shunt; TUG: Timed up and Go test; MMSE, Mini-mental State Examination.

\section{Introduction}

Idiopathic normal-pressure hydrocephalus (iNPH) is a pathological condition attributable to impaired cerebrospinal fluid absorption that gives rise mainly to a gait disturbance as well as to cognitive disorders and urination disorders in the absence of any preceding disease, such as subarachnoid hemorrhage or meningitis, and it is commonly seen in the elderly and slowly progresses. It is a syndrome in which it is possible to achieve an improvement in symptoms by placing an appropriate shunt [1]. Patients with a combination of iNPH and dementia Alzheimer's type (DAT) have been reported [2,3] and there have also been reports of improvement in the dementia not being achieved by shunting alone, even when the gait disturbance had improved $[3,4]$. There have been reports suggesting that donepezil is effective $[3,5]$. Although there were few cases in those reports, they implied that donepezil's efficacy against mild cognitive impairments (MCI or DAT) persisted after lumbar-peritoneal shunt (LPS) placement.

In the present report, four patients who showed improvements of gait disturbances and cognitive functions after LPS placement and donepezil administration are described.

\section{Case report}

The diagnosis of iNPH was made in cases in which there was disproportionately enlarged subarachnoid-space hydrocephalus (DESH), which is seen as enlargement of the subarachnoid space of the basal cistern of the brain and Sylvian fissure, in addition to enlargement of the cerebral ventricles and narrowing of the subarachnoid space of the upper fornix area and longitudinal fissure of the cerebrum on CT and MRI scans (SINPHONI) [1], in which Parkinson's disease and dementia with Lewy bodies had been ruled out by myocardial scintigraphy [6], and that exhibited manifestations such as a gait disturbance, memory impairment, and incontinence.

\begin{tabular}{|c|c|c|c|c|c|c|c|c|}
\hline \multirow{2}{*}{$\begin{array}{l}\text { Case } \\
\text { No. }\end{array}$} & \multirow{2}{*}{$\begin{array}{l}\text { Age } \\
\text { (y) }\end{array}$} & \multirow[b]{2}{*}{ Sex } & \multirow{2}{*}{$\begin{array}{l}\text { MMSE } \\
\text { Pre }\end{array}$} & \multirow[b]{2}{*}{$\begin{array}{l}\text { Post } \\
\text { 1w }\end{array}$} & \multirow[b]{2}{*}{$\begin{array}{l}\text { Post } \\
3 m\end{array}$} & \multirow{2}{*}{$\begin{array}{l}\text { TUG } \\
\text { Pre }\end{array}$} & \multirow[b]{2}{*}{$\begin{array}{l}\text { Post } \\
\text { 1w }\end{array}$} & \multirow[b]{2}{*}{$\begin{array}{l}\text { Post } \\
3 m\end{array}$} \\
\hline & & & & & & & & \\
\hline 1 & 77 & $\mathrm{M}$ & 27 & 27 & 29 & 20 & 17 & 15 \\
\hline 2 & 77 & $\mathrm{~F}$ & 29 & 24 & 30 & 22 & 17 & 15 \\
\hline 3 & 72 & $\mathrm{~F}$ & 23 & 28 & 30 & 22 & 14 & 14 \\
\hline 4 & 81 & $M$ & 29 & 27 & 30 & 16 & 14 & 12 \\
\hline
\end{tabular}

Table 1: Comparison between the preoperative and postoperative results of the MMSE and TUG of four patients who underwent LPS placement followed by donepezil administration. M, male; F, female; pre, preoperative; post $1 \mathrm{w}, 1$ week after LPS placement; post $3 \mathrm{~m}, 3$ months after LPS placement 
Citation: Moriuchi S, Dehara M, Shimizu T, Fukuya S, Fukunaga T, et al. (2015) Lumbar-Peritoneal Shunt followed by Donepezil Administration for Residual Cognitive Impairment in Idiopathic Normal Pressure Hydrocephalus: A Case Report. J Neurol Neurophysiol 6: 274 . doi: $10.4172 / 2155-9562.1000274$

Page 2 of 3

The subjects were four patients ( 2 men, 2 women), ranging in age from 72 to 81 years (mean: 77 years), who were treated with an LPS in Rinku General Medical Center, Izumisano City, Osaka, Japan, between July 2011 and June 2012 (Table 1). A total of $30 \mathrm{~mL}$ of spinal fluid were removed in the tap test [1], before and after which, the Mini-Mental State Examination (MMSE) and Timed Up and Go Test (TUG) were performed. The indications for surgery were evidence of DESH on the above-mentioned imaging studies, an improvement of $10 \%$ or more on the TUG or MMSE after the tap test, and only patients with a strong desire for surgery. The cases were classified as probable NPH according to the iNPH guidelines (2nd revised edition) [1].

Surgery in every patient consisted of placement of an LPS with a Codman-Hakim programmable valve (CHPV; Siphonguard; Johnson and Johnson, Raynham, MA, USA)[7]. The quick reference table for predicting the initial CHPV pressure based on spinal fluid pressure, height, and weight at the time of the tap test (Miyake formula [8]) was used as the basis for setting the shunt pressure, and when low spinal pressure symptoms, such as orthostatic nausea and dizziness, were severe, the pressure was raised, using improvement in the TUG as an indicator, and when there was no improvement in the TUG, the pressure was lowered further. The changes in pressure were made in 30-mmH2O gradations [1].

In these four cases in which some improvements or declines in cognitive symptoms were observed at a week after LPS placement, donepezil therapy was started with $3 \mathrm{mg}$ once daily, increased to $5 \mathrm{mg}$ once daily one week later, and the results were evaluated using the MMSE at 3 months after LPS placement. Improvements in the gait disturbance or the cognitive symptoms were observed after LPS in comparison with before the shunt was placed (Table 1). There was a $25 \%$ or greater postoperative shortening of the TUG time in all four patients. There were improvements of $25-36 \%$ in the four patients compared with before LPS placement. No hydrops or nontraumatic subdural hematomas, which have been reported as postoperative complications, were observed [9].

Because the memory disorder or loss of activity persisted postoperatively, donepezil therapy was started in these four cases. The mean MMSE score of the four patients was 27 points before surgery and 30 points after donepezil administration for 3 months. In case 3 , the MMSE improved from 23 to 28 , especially in orientation, attention, and calculation, but the memory disturbance continued, which could be seen from the category of recall in the MMSE (Table 2); given a score of 14.8 on the cognitive subscale of the Alzheimer's Disease Assessment Scale (ADAS-cog)(Table 3), DAT was diagnosed preoperatively. After LPS placement, mild cognitive impairment persisted, especially in the category of recall in the MMSE, and donepezil therapy was started one week later. MMSE scores improved to 30 at 3 months after LPS placement. The TUG improved from 22 seconds to 14 seconds postoperatively. The CT scan of Case 3 showed enlargement of the subarachnoid space of the Sylvian fissure, in addition to enlargement of the cerebral ventricles (Figure 1A) and narrowing of the subarachnoid space of the upper fornix area and longitudinal fissure of the cerebrum (Figure 1B) preoperatively, which was thought to indicate DESH. After the LPS were placed, the ventricle shrank, and narrowing of the subarachnoid space improved (Figure $1 \mathrm{C}, 1 \mathrm{D})$.

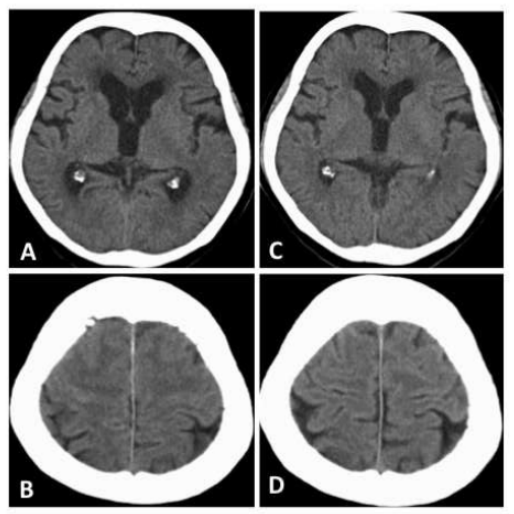

Figure 1: Axial CT scan images of Case 3, preoperative (A, B) and 3 months after LPS placement (C, D).

\begin{tabular}{|l|l|l|l|}
\hline Category (possible points) & Preope & Postope 1w & Postope 3m \\
\hline Orientation to time (5) & 3 & 5 & 5 \\
\hline Orientation to place (5) & 5 & 5 & 5 \\
\hline Registration (3) & 3 & 3 & 3 \\
\hline Attention and calculation (5) & 1 & 5 & 5 \\
\hline Recall (3) & 2 & 1 & 3 \\
\hline Language (2) & 2 & 2 & 2 \\
\hline Repetition (1) & 1 & 1 & 1 \\
\hline Complex commands(6) & 6 & 6 & 6 \\
\hline Total (30) & 23 & 28 & 30 \\
\hline
\end{tabular}

Table 2: Details of MMSE categories in the preoperative and postoperative results of Case 3. preope, preoperative; postope, postoperative; $1 \mathrm{w}$, one week after LPS placement; $3 \mathrm{~m}, 3$ months after LPS placement 
Citation: Moriuchi S, Dehara M, Shimizu T, Fukuya S, Fukunaga T, et al. (2015) Lumbar-Peritoneal Shunt followed by Donepezil Administration for Residual Cognitive Impairment in Idiopathic Normal Pressure Hydrocephalus: A Case Report. J Neurol Neurophysiol 6: 274 . doi: $10.4172 / 2155-9562.1000274$

Page 3 of 3

\begin{tabular}{|l|l|l|}
\hline Category (possible points) & Postope $1 \mathbf{w}$ & Postope 3m \\
\hline Momory and new learning(0-35) & & \\
\hline Word recall & 6 & 5 \\
\hline Orientation & 2 & 1 \\
\hline Word recognition & 0.7 & 1.7 \\
\hline Remembering test & 0 & 0 \\
\hline Language(0-25) & 2 & 0 \\
\hline Praxis(0-10) & 4 & 1 \\
\hline Total $(0-70)$ & 14.7 & 8.7 \\
\hline
\end{tabular}

Table 3: Details of ADAS-cog categories in the postoperative results of Case 3. postope, postoperative; $1 \mathrm{w}$, one week after LPS placement; $3 \mathrm{~m}, 3$ months after LPS placement

\section{Discussion}

Four iNPH patients whose gait disturbances improved after LPS placement and whose cognitive functions improved after administration of donepezil were described. The reason why LPS placement improves cognitive impairment may be as follows. The compression of the fornix by hydrocephalus may have been the cause of the dementia in patients in whom the dementia improved in response to LPS placement alone [10]. Indeed, in a few patients, cognitive symptoms improved as a result of relief of the compression of the fornix by the LPS. In all patients, TUG improved as expected from the preoperative tap test. MMSE did not improve, except in Case 3. Their persistent cognitive impairments improved after the administration of donepezil. All four patients had MCI and could also be diagnosed as having possible DAT, because cognitive impairments continued for several months and were aggravated recently, and persisted even after LPS placement [11]. As seen in Case 3, after LPS placement and release of compression of the fornix or frontal lobe, orientation and attention improved, but memory impairments in the category of recall persisted in the MMSE and ADAS-cog. To treat this cognitive impairment, oral donepezil was started, and cognitive impairments shown in recall on the MMSE and memory on the ADAS-cog improved (Table 2, 3). In conclusion, as in these four patients with iNPH whose cognitive impairments persisted after LPS placement, an improvement in cognitive impairments can be expected with donepezil treatment.

\section{References}

1. Hashimoto M, Ishikawa M, Mori E, Kuwana N; Study of INPH on neurological improvement (SINPHONI) (2010) Diagnosis of idiopathic normal pressure hydrocephalus is supported by MRI-based scheme: a prospective cohort study. Cerebrospinal Fluid Res 7: 18.
2. Bech-Azeddine R, Høgh P, Juhler M, Gjerris F, Waldemar G (2007) Idiopathic normal-pressure hydrocephalus: clinical comorbidity correlated with cerebral biopsy findings and outcome of cerebrospinal fluid shunting. J Neurol Neurosurg Psychiatry 78: 157-161.

3. Hamilton R, Patel S, Lee EB, Jackson EM, Lopinto J, et al. (2010) Lack of shunt response in suspected idiopathic normal pressure hydrocephalus with Alzheimer disease pathology. Ann Neurol 68: 535-540.

4. Koivisto AM, Alafuzoff I, Savolainen S, Sutela A, Rummukainen J, et al. (2013) Poor cognitive outcome in shunt-responsive idiopathic normal pressure hydrocephalus. Neurosurgery 72: 1-8.

5. Ferris SH, Farlow M (2013) Language impairment in Alzheimer's disease and benefits of acetylcholinesterase inhibitors. Clin Interv Aging 8: 1007-1014.

6. Nagayama H, Hamamoto M, Ueda M, Nagashima J, Katayama Y (2005) Reliability of MIBG myocardial scintigraphy in the diagnosis of Parkinson's disease. J Neurol Neurosurg Psychiatry 76: 249-251.

7. Mori K (2001) Management of idiopathic normal-pressure hydrocephalus: a multiinstitutional study conducted in Japan. J Neurosurg 95: 970-973.

8. Miyake H, Kajimoto Y, Tsuji M, Ukita T, Tucker A, (2008) Development of a quick reference table for setting programmable pressure valves in patients with idiopathic normal pressure hydrocephalus. Neuro Med Chir (Tokyo) 48: 427-432.

9. Bergsneider M, Miller C, Vespa PM, Hu X (2008) Surgical management of adult hydrocephalus. Neurosurgery 62 Suppl 2: 643-659.

10. Hattori T, Sato R, Aoki S, Yuasa T, Mizusawa H (2012) Different patterns of fornix damage in idiopathic normal pressure hydrocephalus and Alzheimer disease. AJNR Am J Neuroradiol 33: 274-279.

11. McKhann GM, Knopman DS, Chertkow H, Hyman BT, Jack CR Jr, et al. (2011) The diagnosis of dementia due to Alzheimer's disease: recommendations from the National Institute on Aging-Alzheimer's Association workgroups on diagnostic guidelines for Alzheimer's disease. Alzheimers Dement 7: 263-269. 